\title{
AAV-Mediated In Vivo CAR Gene Therapy for Targeting Human T Cell
}

\section{Leukemia}

3

4

5

6

Waqas Nawaz ${ }^{1,2,3}$, Bilian Huang ${ }^{1,2,3}$, Shijie $\mathrm{Xu}^{1,2,3}$, Yanlei $\mathrm{Li}^{4}$, Linjing $\mathrm{Zhu}^{5}$, Zhiwei $\mathrm{Wu}^{1,2,3^{*}}$ and Xilin $\mathrm{Wu}^{1,2,3^{*}}$

1. Center for Public Health Research, Medical School, Nanjing University, Nanjing,

People's Republic of China.

2. State Key Laboratory of Analytical Chemistry for Life Science, Nanjing University, Nanjing,

People's Republic of China.

3. Jiangsu Key Laboratory of Molecular Medicine, Medical School, Nanjing University, Nanjing,

People's Republic of China.

4. Y-Clone Medical Science Co. Ltd. Suzhou, People's Republic of China.

5. Abrev Biotechnology, Nanjing, People's Republic of China.

Short title: Development of off-the-shelf CAR gene therapy

Keywords: Adeno-associated Virus, AAV delivering CAR gene therapy, CAR-T therapy, T cell leukemia, AAV-CD4+ CAR cells, Off the shelf CAR cells development, Immunotherapy.

*Corresponding author: Zhiwei Wu, E-mail: wzhw@nju.edu.cn and Xilin Wu, E-mail:

xilinwu@nju.edu.cn; Mailing address: Center for Public Health Research, Medical School,

Nanjing University, 22\# Hankou Road, Nanjing, Jiangsu Province, 210093, China.

Phone: 86 (25) 8368-6092. Fax: 86 (25) 8359-6023. 


\section{Abstract}

Chimeric antigen receptor (CAR) T cell therapy is the most active field in immuno-oncology and brings substantial benefit to patients with B cell malignancies. However, the complex procedure for CAR $\mathrm{T}$ cell generation hampers its widespread applications. Here, we describe a novel approach in which human CAR T cells can be generated within the host upon injecting an Adenoassociated virus (AAV) vector carrying the CAR gene, which we call AAV delivering CAR gene therapy (ACG). Upon single infusion into a humanized NCG tumor mouse model of human T cell leukemia, AAV generates sufficient numbers of potent in vivo CAR cells, resulting in tumor regression; these in vivo generated CAR cells produce antitumor immunological characteristics. This instantaneous generation of in vivo CAR $\mathrm{T}$ cells may bypass the need for patient lymphodepletion, as well as the ex vivo processes of traditional CAR T cell production, which may make CAR therapy simpler and less expensive. It may allow the development of intricate, individualized treatments in the form of on-demand and diverse therapies.

\section{Significance Statement}

AAV can generate enough CAR cells within the host. That act as a living drug, distributed throughout the body, and persist for weeks, with the ability to recognize and destroy tumor cells. 


\section{Introduction}

56 Chimeric antigen receptor (CAR) $\mathrm{T}$ cells have achieved significant clinical recognition with

57 remarkable responses documented for autologous CD19 (a B cell-specific antigen) redirected T

58 cells for the treatment of patients with relapsed or refractory B cell malignancies [1]. In 2017, the

59 US Food and Drug Administration (FDA) approved two autologous CD19-CAR T cell products

60 to treat certain types of relapsed or refractory large B cell lymphoma and acute lymphoblastic

61 leukemia [2]. However, CAR T cell therapy still faces skepticism and has plenty of room for

62 improvement, including challenges ranging from understanding the mechanisms and management

63 of innate and acquired resistance and cytokine release syndrome (CRS) to neurologic toxicity and

64 establishment of more efficient, less expensive, and quicker mechanisms of manufacturing [3].

65 Current clinical-scale manufacturing of $\mathrm{T}$ lymphocytes involves complex processes of isolation,

66 genetic modification, and selective expansion of redirected cells ex vivo prior to their infusion into

67 patients. This makes the manufacturing process complicated, costly and associated with clinical

68 hazards. Thus, the manufacturing of safe, affordable, easier and effective CAR T cells is critical

69 for making this therapy widely accessible for a larger population [4]. Moreover, these complicated

70 procedures are resource-intensive and require substantial technical expertise and can only be

71 performed at a limited number of specialized centers worldwide [5]. The most crucial step to

72 overcome these limitations is to create novel strategies for making this complicated and risky cell

73 therapy affordable, safer and customizable. This may in turn enable CAR T cells to outcompete

74 chemotherapy as front-line therapy.

75 Adeno-associated virus (AAV), a small, icosahedral nonenveloped virus of $25 \mathrm{~nm}$ in diameter

76 containing a single-stranded DNA genome, has the potential to solve this problem. AAV has

77 become an essential therapeutic gene delivery vector in recent years, it is frequently used to deliver 
genetic material into target cells to cure or treat disease, and is extensively used in clinical applications in academia and industry [6]. To date, almost 200 clinical trials using AAV have been completed or are ongoing [7].

81 To manipulate immune cells in vivo, the carrier needs to be taken up by cells and import the 82 encoding DNA into the nucleus. Previous studies showed that AAV could transduce both dividing 83 and non-dividing cells with superior infection efficiency, which enables AAV to be widely 84 applied in gene therapy in vivo [8]. In our current study, we choose AAV-DJ (type2/type8/type9 85 chimera), which was developed by using DNA shuffling technology to produce hybrid capsids 86 from eight distinct wild-type AAV [9]. Previously, we reported AAV-DJ to deliver antibody gene 87 in vivo, where antibodies were persistent to express in cells for more than 9 weeks [10]. The 88 capability to transduce different cell types is mainly determined by the AAV capsid proteins. 89 AAV-DJ capsid was selected [9], which has higher tropism and extremely robust at transducing 90 cells $[9,11]$.

91 We hypothesize that human CAR T cells can be generated to specifically kill target cells within

92 the host upon injecting an AAV vector carrying the CAR gene, which we call AAV delivering

93 CAR gene therapy (ACG). To prove this concept, we furnished an AAV vector with plasmid DNA

94 encoding a CD4-CAR, which is composed of a single-chain antibody (scFv) specific for the

95 extracellular domain of the CD4 antigen fused with CD28, 4-1BB, and CD3 zeta cytoplasmic

96 signaling domains. We demonstrated that the modified AAV vector could safely deliver the CAR

97 gene into the host cells of humanized NCG (NCG-HuPBL) mice and reprogrammed cells to 98 express the CAR. As envisioned, the AAV vector generated enough CAR T cells in vivo and thus 99 caused effective tumor regression and produced antitumor immunological characteristics. In 100 addition, ex vivo experiments indicated that our AAV delivering the CD4-CAR potently eliminated 
101 tumor cells from adult $\mathrm{T}$ cell leukemia (ATL). The current approach may skip the technically

102 challenging ex vivo procedures and enable the host to create its CAR T cells.

\section{Methods and materials}

105 Mice

106 NCG mice were purchased from GemPharmaTech. NCG mice were maintained in accordance

107 with the Guide for the Care and Use of Laboratory Animals of the Medical School of Nanjing

108 University. All experiments were performed according to the guidelines of the Institutional Animal

109 Committee of Nanjing University.

\section{$110 \quad$ PBMCs and cell lines}

111 Peripheral blood mononuclear cells (PBMCs) (derived from healthy donors visiting the Drum

112 Tower Hospital, Nanjing University) were isolated from buffy coats by Ficoll-Hypaque gradient

113 separation, and cultured in RPMI-1640, 10\% heat-inactivated FBS and freshly added recombinant

114 human interleukin-2. Excess PBMCs were cryopreserved until use. For stimulation, PBMCs were

115 washed once in T cell medium without IL-2 and then suspended at a concentration of $10^{6} / \mathrm{ml}$ in T

116 cell medium containing IL-2 (300 IU/ml) and anti-human CD3 antibody $(1 \mu \mathrm{g} / \mathrm{ml})$. Cells were

117 incubated in $5 \% \mathrm{CO}_{2}$ at $37^{\circ} \mathrm{C}$ for 2 days until $\mathrm{AAV}$ transduction.

118 AAV-293T (Thermo Fisher) and HEK-293T (ATCC) cells were maintained in Dulbecco's

119 modified Eagle medium (DMEM) containing 10\% FBS and 2 mM glutamate. H929, MT2, and

120 Jurkat cell lines (ATCC) were grown in RPMI-1640 with 10\% heat-inactivated FBS. CD4

121 expression was authenticated by flow cytometry in MT2 and Jurkat cell lines prior to experiments.

122 All cell culture media contained $100 \mathrm{U} / \mathrm{ml}$ penicillin-streptomycin (Gibco, Invitrogen). All cell

123 lines were maintained in an environment of $37^{\circ} \mathrm{C}$ and $5 \% \mathrm{CO}_{2}$. 


\section{Construction of AAV-CAR vectors}

125 A recombinant AAV-2 ITR-containing plasmid (pAAV-MCS) with an expression cassette 126 containing inverted terminal repeats was used for AAV generation. To generate AAV-CD20CAR 127 and AAV-CD4CAR, we used the sequence of CD20-binding scFv from NCBI (GenBank, 128 KM043780) and the sequence of Hu5A8 specific for the CD4 backbone with a 20-mer (Gly4Ser) 129 adopted from our previous study [10]. The scFv was fused with CD28 hinge-transmembrane 130 regions linked to 4-1BB (CD137) intracellular domains and the CD3 zeta intracellular domain 131 [12]. CAR DNA (Hu5A8-CD28-41BB-CD3 or Rit-CD28-41BB-CD3) was cloned into the

132 ITR/MCS-containing vector. Plasmid sequence and construction were confirmed by sequence 133 analysis and restriction enzymes. Stocks of pAAV-Hu5A8-CD28-41BB-CD3 and pAAV-Rit134 CD28-41BB-CD3 were prepared as pAAV vectors of interest (VOIs).

\section{AAV production}

136 AAV of DJ subtype was prepared by polyethyleneimine (PEI)-based triple transfections of pAAV-

137 VOI, pHelper (helper plasmid) and pAAV-DJ (catalog number VPK-430-DJ, Cell Biolabs), as 138 described [13]. Briefly, the medium of 80-90\% confluent AAV-293T cells in $15 \mathrm{~cm}$ plates was 139 changed to fresh DMEM 2 hours before transfection. Plasmids at a ratio of 1:1:1 (25 $\mu \mathrm{g}$ each 140 plasmid for a $15 \mathrm{~cm}$ plate) were mixed with $2 \mathrm{ml}$ Opti-MEM in a $15 \mathrm{ml}$ tube. Three hundred 141 microliters of PEI (Polysciences Inc.) (1 mg/ml) was added to $2 \mathrm{ml}$ Opti-MEM in another $15 \mathrm{ml}$ 142 tube and was mixed by vortexing and incubated for $15 \mathrm{~min}$ at room temperature, after which the 143 mixture was gently added to the serum-free medium in the plate and incubated in a standard 5\%

$144 \mathrm{CO}_{2}$ and $37^{\circ} \mathrm{C}$ incubator. The supernatant was collected 24, 48, 72, and 96 hours posttransfection, 145 and the debris was cleared by centrifugation and filtration $(0.45 \mu \mathrm{M})$. AAV was concentrated by 146 centrifuging (1000g for 5min) with $10 \%$ polyethylene glycol 8000 and $14 \% 4 \mathrm{M} \mathrm{NaCl}$ (by volume) 
147 and incubated overnight at $4^{\circ} \mathrm{C}$. The next morning, the sample was transferred to $50 \mathrm{ml}$ conical

148 tubes and centrifuged at $4000 \mathrm{~g}$ for $30 \mathrm{~min}$ at $4^{\circ} \mathrm{C}$. The AAV pellet was resuspended in PBS and

149 stored at $-80^{\circ} \mathrm{C}$. We used qPCR to determine the number of genome-containing particles in an

150 AAV prep using SYBR green technology, adopted from our previously reported protocol [10].

151 The detailed procedure of determining the genome-containing particles in an AAV prep using

152 SYBR green technology is explained in the supplementary information.

\section{AAV transduction of HEK-293T and T cells.}

154 Three different doses of AAVs were administered: 1x10 4 AVs/cell was considered AAV low

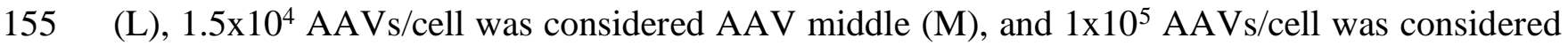

156 AAV high $(\mathrm{H})$. The day before transduction, HEK-293T cells were trypsinized and counted, and

$157 \quad 1-4 \times 10^{5}$ cells were plated in 2.0-3.0 ml complete culture medium and incubated at $37^{\circ} \mathrm{C}$ overnight.

158 Sixteen hours later, purified AAV (AAV-CD4CAR or AAV-CD20CAR) was added to each well

159 at different doses by gentle swirling/mixing. Twenty-four hours post-transduction, fresh media

160 containing $20 \%$ FBS was added, and the cells were cultured for 2-3 days and then analyzed by

161 FACS, or fluorescence microscopy.

162 The T cells were activated and infected with AAV-CAR as described [14]. Briefly, $10^{6}$ cells were

163 plated on day 0 and activated for two days in the presence of $300 \mathrm{IU} / \mathrm{ml} \mathrm{IL-2}$ and $1 \mu \mathrm{g} / \mathrm{ml}$ anti-

164 human CD3 antibody. On day 2, purified AAV (AAV-CD4CAR or AAV-CD20CAR) was added

165 to each well at different doses by gentle swirling/mixing and incubated at $37^{\circ} \mathrm{C}$ in the presence of

$1665 \% \mathrm{CO}_{2}$. The next morning following transduction, fresh media containing $20 \% \mathrm{FBS}$ was added,

167 and the cells were cultured for 2-3 days and then analyzed by FACS or florescence microscopy. 


\section{Flow cytometry}

169 All antibodies for flow cytometry were purchased from BioLegend or Invitrogen. Cells were

170 stained with target antibodies (according to the experiment's requirement), whereas for CAR

171 expression, target antigen (CD4Fc protein) was used. After 30 minutes at $4^{\circ} \mathrm{C}$, cells were washed

172 once, suspended in FACs buffer, and stained with Alexa flour488 anti-rabbit for 30 minutes at

$1734^{\circ} \mathrm{C}$. For in vivo samples, cells were lysed by RBC lysis buffer (BD), by incubating the cells with

$1741 \mathrm{X}$ lysis buffer at room temperature for 10 minutes. Labeled cells were washed twice and 175 suspended in FACs buffer. All cells were measured and sorted by using a NovoCyte FACS, and 176 analyzed with FlowJo software version 10.2.

\section{CAR T cell-mediated cytotoxicity assay using CFSE and 7-AAD}

178 Cytotoxicity assays were carried out using CFSE and 7-AAD, as previously described [15].

179 Briefly, CFSE-labeled target cells were incubated with different doses of AAV-CD4CAR-and 180 AAV-CD20CAR-infected PBMCs for $24 \mathrm{hrs}$ at $37^{\circ} \mathrm{C}$. After 24 hours of incubation, 7-amino181 actinomycin D (7-AAD; BD Pharmingen) was added, as recommended by the manufacturer. The 182 fluorescence was analyzed by flow cytometry. Target cell cytotoxicity was calculated using the 183 following formula: cytotoxicity $=100 \times[($ CFSE-labeled dead target cells) $/$ (CFSE-labeled dead 184 target cells + CFSE-labeled lived target cells)]

185 In vivo NCG-HuPBL mouse model for the in vivo $\mathrm{CD3}^{+} \mathrm{CD4}^{+}$depletion assay

186 NCG mice were purchased from GemPharmaTech. NCG mice were maintained in accordance 187 with the Guide for the Care and Use of Laboratory Animals of the Medical School of Nanjing 188 University. All experiments were performed according to the guidelines of the Institutional Animal 189 Committee of Nanjing University. The humanized NCG (NCG-HuPBL) mouse model was 190 developed as reported previously by our group [16]. Briefly, human PBMCs were obtained from 
191 healthy individuals' peripheral venous blood and purified by Ficoll-Paque (Pharmacia, Piscataway,

192 NJ) density gradient centrifugation as described above in detail. Freshly isolated PBMCs were

193 resuspended in PBS, and $1.5 \times 10^{7} \mathrm{PBMC} /$ mouse were injected intraperitoneally into NCG mice.

194 PBMC engraftment was confirmed after 3 weeks by FACS, and mice with an appropriate percent

195 ratio of $\mathrm{CD} 3+\mathrm{CD} 4+$ and $\mathrm{CD} 3+\mathrm{CD} 8+$ were considered successful NCG-HuPBL mouse models. A

196 total of $5 \times 10^{6}$ ex vivo-transduced AAV-CD4CAR T cells or $1 \times 10^{11}$ AAV-CD4CAR T cells and

$1971 \times 10^{11} \mathrm{AAV}-\mathrm{CD} 20 \mathrm{CAR} \mathrm{T}$ cells were injected into each Hu-NCG mouse according to the specified

198 grouping. Mice were bled via the submandibular vein, and peripheral blood cell populations and

199 CAR expression were analyzed periodically using flow cytometry.

\section{In vivo NCG-HuPBL tumor mouse model}

201 After successfully engrafting PBMCs in NCG mice, we modeled T cell tumors by intraperitoneally

202 injecting $2.5 \times 10^{6}$ luciferase-expressing MT2 cells into each NCG-HuPBL mouse (generation of

203 stable luciferase-expressing MT2 ATL tumor cell line is explained in Supplementary information).

204 NCG-HuPBL mice injected with luciferase-expressing MT2 cells underwent in vivo

205 bioluminescence imaging at various times as specified for each experiment. Luciferase-based

206 bioluminescence imaging was performed with a NightOWL II LB 983 (Berthold) in vivo imaging

207 system. Mice were first anesthetized by inhaling isoflurane and were maintained with $2 \%$

208 isoflurane during imaging procedures. Mice were injected (I.P.) with D-luciferin (prepared in

209 DPBS) at $150 \mathrm{mg} / \mathrm{kg}$ and imaged immediately after anesthesia. Images were captured, and

210 bioluminescence intensity and tumor area were quantitated using Indigo live imaging analysis

211 (Berthold). Total flux and area were measured through the automated analysis method by Indigo

212 (Berthold). 


\section{Confocal microscopy}

214 Samples from mice for confocal imaging were prepared as described previously [17]. Briefly, cells

215 were stained for CD4-Fc protein and with an anti-CD45 ${ }^{+}$antibody for 1 hour. Next, the cells were

216 washed and stained with anti-CD4-Fc secondary antibody for 1 hour. Following washing, the cells

217 were transferred onto a fibronectin matrix (Sigma Aldrich) and fixed in 2\% paraformaldehyde.

218 Slides were mounted with \#1.5 coverslips and Mowiol mounting medium and examined using an

219 FV3000 confocal microscope.

\section{$220 \quad$ In vivo cytokine measurement}

221 In vivo cytokines from the serum/plasma of mice were measured using the Cytometric Bead Array

222 Human Th1/Th2 Cytokine Kit (BD), following the manufacturer's instruction. The kit was used

223 for the simultaneous detection of Human TNF, IL-2, IL-6, IL-4, and IL-10 in a single sample. The

224 Cytometric Bead Array Human Th1/Th2 Cytokine Kit contains a mixture of various capture beads

225 with different fluorescent intensities which are coated with capture antibodies specific for each

226 cytokine. First, we mixed the beads coated with six specific capture antibodies. Next, $50 \mu \mathrm{L}$ of the

227 mixed captured beads, $50 \mu \mathrm{L}$ of phycoerythrin (PE) detection reagent, and $50 \mu \mathrm{L}$ of the unknown

228 serum sample or standard dilutions were added sequentially to each assay tube and incubated for

$2292 \mathrm{~h}$ at room temperature in the dark. The samples were washed with $500 \mu \mathrm{L}$ of wash buffer and

230 centrifuged at $800 \mathrm{~g}$ for 5 mint. The bead pellet was resuspended in $400 \mu \mathrm{L}$ buffer after discarding

231 the supernatant. All the samples were sorted on the NovoCyte FACS. Fluorescent intensities give

232 the individual cytokine concentration. The standard curve for the CBA assay was determined using

233 different dilutions. The data were analyzed with FCAP Array software provided by BD.

\section{Statistical analysis}


236 Specific statistical tests and metrics (median, mean, standard error) used for comparisons, along

237 with sample sizes, are described in the Results and Figure legends. All statistical analyses were

238 performed using GraphPad Prism software version 6.0. ns, no significance; ****P $<0.0001$; ***,

$239 \mathrm{P}<0.001 ; * *, \mathrm{P}<0.01 ; *, \mathrm{P}<0.05$.

241 Results

\section{Design and characterization of AAV-CD4CAR}

As schematically shown in Figure 1a, AAV-CD4CAR is the third-generation CAR construct

245 composed of anti-CD4 scFv, hinge $(\mathrm{H})$ and transmembrane regions, and $\mathrm{CD} 28$ and 4-1BB

246 intracellular signaling domains in tandem with the CD3 zeta signaling domain. The CD4-CAR

247 DNA molecule was subcloned into the pAAV-VOI plasmid, which was mixed with the plasmids

248 pAAV-Helper and pAAV-DJ to generate AAV virus carrying the CD4-CAR gene (AAV-

249 CD4CAR). To verify the AAV-CD4CAR construct, infected HEK-293T cells were subjected to

250 Western blot and fluorescence-activated cell sorting (FACS) analysis. Immunoblotting with an

251 anti-CD3 zeta monoclonal antibody showed bands of the expected size for the AAV-CD4CAR

252 CD3 zeta fusion protein, while the control cells infected with AAV-GFP showed no bands (Fig.

253 1b). Furthermore, AAV-CD4CAR infection efficiency was analyzed through FACS using CD4-

254 Fc protein (Fig. 1c). Moreover, AAV-CD4CAR infects cells with limited toxicity and high

255 viability (Supplementary Fig. 1a-c).

256 We characterized the effect of AAV-CD4CAR on T cells from healthy donors. Human T cells

257 were activated with IL-2 and anti-human CD3 antibody and then infected with different doses of

258 AAV-CD4CAR (MOI $1 \times 10^{4}, 0.5 \times 10^{5}$, and $\left.1 \times 10^{5}\right)$. As expected, a dose-dependent expression

259 of the CD4-CAR on T cells was observed (Figure 1d). We found that AAV-CD4CAR induced 
dose-dependent expression in three different healthy donor samples. However, the percentage of

261 expression varied among donors, but the difference was not statistically significant

262 (Supplementary Figure 2a). Upon more detailed phenotyping, we found that CD4+ T cells also

263 expressed AAV-CD4CAR but at a significantly lower rate than CD8+ T cells (Supplementary

264 Figure 2b).

265 In summary, these results confirmed that our AAV-CD4CAR contain the CD3 zeta on the

266 intracellular end and the anti-CD4 scFv on the extracellular end, verifying that all other elements

267 were present. Furthermore, AAV-CD4CAR infect the T cells and produce AAV-CD4CAR T cells

268 in a dose-dependent manner.

269 AAV-CD4CART cells are CD4 specific and eliminate leukemic cells in vitro and aggressive 270 CD4 $^{+}$ATL cells ex vivo

271 We furnished our AAV-CD4CAR with the scFv region of the Hu5A8 antibody specific for CD4,

272 which we reported previously for HIV treatment [10]. We next evaluated the functional ability of

273 AAV-CD4CAR to eliminate $\mathrm{CD}^{+}$cells in PBMCs from various donors $(\mathrm{n}=10)$ during $\mathrm{T}$ cell

274 expansion following AAV-CD4CAR transduction. As expected, the groups treated with AAV-

275 CD4CAR had a decreased $\mathrm{CD}^{+} \mathrm{CD}^{+} \mathrm{T}$ cell subset within 2-3 days following AAV-CD4CAR

276 transduction in a dose-dependent manner compared to the vector control group (Figure. 2b).

278 AAV-CD4CAR was tested in vitro for its antileukemic activity using CD4-positive MT2 and

279 Jurkat cells as targets. In addition, the CD4-negative cell lines H929 and AAV-CD20CAR served

280 as a negative control. The MT2 cell line is an adult T cell leukemia (ATL) cell line with aberrant

281 CD4 expression established by co-culture of normal human cord leukocytes with leukemic cells

282 from ATL patients [18]. Jurkat cells are immortalized T lymphocytes obtained from a patient with

283 T cell leukemia [19]. CD4 expression on MT2 and Jurkat cells was counterchecked by flow 
cytometry prior to experiments. H929 is a B cell line with CD38 expression established from a malignant effusion in a patient with myeloma [20]. 48 hours after co-culture with human PBMCs infected with various doses of AAV-CD4CAR, MT2, and Jurkat cells were effectively lysed in a

287 dose-dependent manner. However, neither the CD4- cell line (H929) nor the MT2 cells treated

288 with a CD20-CAR were lysed (Figure. 2c). Next, we performed an ex vivo experiment to evaluate 289 the ability of AAV-CD4CAR to kill primary ATL cells. CD3 ${ }^{+} \mathrm{CD} 4{ }^{+}$primary cells from an 290 unclassified and chemotherapy-resistant ATL patient were isolated ex vivo and served as a target 291 population. $\mathrm{CD}^{+}{ }^{+} \mathrm{CD} 4{ }^{+}$ATL cells were completely lysed (depleted to $~ 0 \%$ ) by AAV-CD4CAR 29248 hours post-infection, while AAV-CD20CAR was unable to lyse CD3 ${ }^{+}$CD4 ${ }^{+}$ATL cells (Figure. 293 2d). In summary, these results show that AAV-CD4CAR-infected cells exhibited dose-dependent, 294 profound, and specific antitumor activity against T cell leukemia cell lines. Similarly, they could 295 also completely lyse the tumorigenic $\mathrm{CD}^{+} \mathrm{CD}^{+}$population in samples from ATL patients in $e x$ 296 vivo coculture. Altogether, these results signify that AAV-CD4CAR-infected cells are specific for $297 \mathrm{CD}^{+}$cells.

298 CAR T cells generated from in vivo reprogramming of lymphocytes via AAV and their antitumor immunological characteristics

300 We hypothesized that our AAV carrying CAR gene construct could reprogram lymphocytes and 301 are able to generate in vivo CAR T cells to kill target cells in a process that we called ACG. To 302 experimentally prove our ACG concept, we transplanted human PBMCs into NCG mice, and after 303 successful engraftment of PBMCs, we injected $1 \times 10^{11}$ AAV-CD4CAR into NCG-HuPBL mice. 304 Interestingly, FACS analysis of blood collected after 48 hours showed that the AAVs started 305 infecting the immune cells, and $9 \%$ of $\mathrm{CD}^{4} 5^{+}$cells expressed the CD4-CAR (Figure. 3a). We 
further confirmed CD4-CAR expression on $\mathrm{CD} 45^{+}$cells through confocal microscopy. Infused AAV-CD4CAR were taken up by lymphocytes, and the CD4-CAR could be observed on the

308 lymphocyte membrane (Figure. 3a).

309 CAR T cells combine features of cell therapy, gene therapy, and immunotherapy. Antitumor 310 characteristics, including transduction efficiency, transgene expression levels, stability or 311 retention, cytokine production, are all key to these processes. Next, we evaluated the stability and 312 biodistribution of AAV-CD4CAR T cells in NCG-HuPBL mice. Our results showed that the 313 AAVs efficiently delivered CD4-CAR DNA into host $\mathrm{CD}^{+} \mathrm{CD} 8^{+} \mathrm{T}$ cells, which resulted in the 314 production of enough CD4-CAR T cells, which circulate in the host body for weeks (Figure. 3b).

315 We quantified the biodistribution of AAV-CD4CAR T cells in different organs after injecting 316 AAV-CD4CAR into NCG-HuPBL mice. AAV-CD4CAR T cells were distributed in various 317 organs, with the highest concentrations in the spleen and blood (Figure. 3c). Cytokine production 318 is a hallmark of CAR T cell therapy; therefore, we assessed cytokine production in an ATL NCG-

319 HuPBL mouse model. Our results showed that in vivo-generated AAV-CD4CAR cells were 320 robustly functional against tumor cells and were able to produce a series of cytokines (Figure. 3d).

321 Taken together, the findings from these experiments suggest that an AAV encoding a CAR gene 322 can reprogram sufficient immune effector cells to generate in vivo CAR T cells. Furthermore, the 323 in vivo-generated AAV-CD4CAR T cells can circulate in the host body for weeks and induce 324 antitumor immunological characteristics. These results indicate that the ACG strategy can generate 325 CAR T cells in vivo.

\section{Functional characterization of in vivo-generated AAV-CD4CAR T cells}

327 Encouraged by the expression and antitumor immunological characteristics of in vivo-generated

328 AAV-CD4CAR T cells, we next decided to compare the functional ability by comparing the 
$\mathrm{CD}^{+}{ }^{+} \mathrm{CD} 4^{+}$cell depletion and CAR expression of in vivo-generated AAV-CD4CAR T cells with

330 those of conventional CAR T cells. We prepared NCG-HuPBL mice transplanted with PBMCs

331 (Figure. 4a) and divided them into four groups: PBS group (untreated control), AAV-CD20CAR

332 group (negative control), AAV-CD4CAR T cell group (positive control), and AAV-CD4CAR

333 group (treatment group). Prior to any treatment, NCG-HuPBL mice were randomly divided into

334 each group with no significant difference in $\mathrm{CD} 3^{+} \mathrm{CD} 4^{+}$percentage at day 0 (Supplementary

335 Figure. 3a). We treated the positive control mice with six million T cells infected in vitro with

336 AAV-CD4CAR. This amount corresponds to the approved high dose of CAR T cells currently

337 being used in clinics, in which patients are administered $2 \times 10^{6} \mathrm{CAR} \mathrm{T}$ cells $/ \mathrm{kg}$ of mean body

338 weight [21]. However, we infused a single dose of $1 \times 10^{11}$ AAV into the treatment group (AAV-

339 CD4CAR) and a negative control group (AAV-CD20CAR). As envisioned, a single dose of AAV

$340\left(1 \times 10^{11}\right)$ infusion was able to yield enough AAV-CD4CAR T cells in vivo, similar to the number

341 of ex vivo transduced cells after infusion (Figure. $3 \mathrm{~b}$ and $3 \mathrm{c}$ ). Correspondingly, $\mathrm{CD} 3^{+} \mathrm{CD} 4^{+}$cells

342 started decreasing within the first week in the positive control and treatment groups. After two

343 weeks, there was a significant difference in $\mathrm{CD} 3{ }^{+} \mathrm{CD} 4^{+}$cell percentage between the positive

344 control and treatment group compared to the negative control and untreated mice (Figure. 4d).

345 However, there was no difference observed between the positive control and the treatment group,

346 suggesting that the targeting potential of the in vivo-generated AAV-CD4CAR T cells was similar

347 to that of the conventional CAR T cells. Similarly, no significant difference was observed in

348 percent survival between the positive control mice and the treatment group (Figure. 4e). In

349 summary, the above results show that in vivo-generated AAV-CD4CAR T cells are functionally

350 potent and effective against the target in NCG-HuPBL mice and have similar efficiency to that of

351 conventionally manufactured CAR T cells. 
352 Next, we also evaluated the relative effects of the ACG approach on blood count, serum chemistry,

353 and mean body. We calculated the bodyweight at different time intervals and collected blood for

354 complete blood count (CBC) and serum chemistry. There was no difference in the mean body

355 weight between the in vivo-generated AAV-CD4CAR $\mathrm{T}$ group and the untreated group

356 (Supplementary Figure. 3b). Furthermore, serum chemistry and complete blood count values were

357 normal and almost similar to those of the untreated mice (Supplementary Figure. 3c, d). The slight

358 difference in the AAV treated group compared to the PBS group may be due to cytokines

359 production in AAV treated groups. In summary, these results suggesting the safety of the ACG

360 approach for the generation of in vivo CAR T cells.

\section{In vivo antitumor activity of ACG}

362 We further assessed whether the in vivo-generated AAV-CD4CAR T cells could eradicate the 363 tumor in NCG-HuPBL mice. To establish a tumor-bearing NCG-HuPBL mouse model, we first 364 injected PBMCs into NCG mice and selected successful PBMC-engrafted NCG-HuPBL mice,

365 followed by intraperitoneal injection of luciferase-expressing MT2 ATL cells to develop an ATL

366 NCG-HuPBL mouse model. Mice were randomly divided into two groups: the untreated group

367 (PBS) and the treated group (AAV-CD4CAR). Two days after establishing the ATL NCG-HuPBL

368 mouse model, we injected $2 \times 10^{11}$ AAVs carrying the CD4-CAR gene into the treatment group

369 (Figure. 5a). Interestingly, the ACG strategy started causing tumor reduction at day 10, which

370 became more apparent at day 17 and day 25 (Figure. 5b, c). The mean tumor area of AAV-

371 CD4CAR-treated mice was significantly reduced compared with that of the untreated group

372 (Figure. 5d). Four out of six mice in the treated group presented complete tumor remission. One

373 mouse (M10) showed tumor recurrence when analyzed at day 25 (Figure. 5b). In the untreated

374 group, none of the mice showed any regression in tumor burden, suggesting stable tumor growth 
over time (Figure. 5b). As CD45+CD4+ cells are present in both healthy PBMCs and MT2 ATL

376 cells, we next analyzed the $\mathrm{CD} 45^{+} \mathrm{CD} 4^{+}$population in mice at the end of the study (day 25 ) to

377 evaluate the tumor burden at the cellular level. Our results showed that the percentage of

$378 \mathrm{CD} 45^{+} \mathrm{CD} 4^{+}$cells in the untreated NCG-HuPBL ATL mice was higher than that in the untreated

379 NCG-HuPBL mice (NCG mice engrafted with PBMCs only, no tumor) (Figure. 5e). This higher

380 percentage of $\mathrm{CD} 45^{+} \mathrm{CD} 4^{+}$cells was due to the injected tumor cells that expressed $\mathrm{CD} 4^{+}$cells.

381 Interestingly, the FACS results also showed that the $\mathrm{CD} 45^{+} \mathrm{CD} 4^{+}$cells were completely depleted

382 in the ACG-treated mice, while no depletion was observed in the untreated mice (Figure. 5f).

383 Furthermore, a significant percentage of $\mathrm{CD}^{+}{ }^{+} \mathrm{CD} 8^{+}$cells showed AAV-CD4CAR expression in

384 the treated group (Figure. 5f). The $\mathrm{CD} 45^{+} \mathrm{CD} 4^{+}$depletion and CAR expression in the $\mathrm{CD} 3^{+} \mathrm{CD} 8^{+}$

385 population agreed with the tumor burden analyzed by IVIS signal and tumor size in each mouse

386 (Supplementary Figure. 4 a, b). Next, we evaluated the tumor burden at the organelle level. Two

387 mice from each group were euthanized at the end of the study, dissected, and analyzed for IVIS

388 signals (Supplementary Figure. 4 c). Tumors were almost completely eradicated from all organs

389 in AAV-CD4CAR-treated mice, except in one mouse (M10), which showed a small tumor burden

390 in the intestinal region (the same mouse that had tumor recurrence). However, a significant amount

391 of tumor was observed in all the organs in the untreated mice (Figure. $5 \mathrm{~g}$ ). In summary, our results

392 showed that when an AAV carrying the CD4-CAR gene was infused into mice, it could quickly

393 reprogram lymphocytes and generate sufficient CAR cells to induce ATL regression in NCG-

394 HuPBL mice, suggesting that ACG could be a potential strategy for tumor treatment. 


\section{Discussion}

AAV has been intensively investigated for gene therapy in preclinical and clinical studies. AAV vectors are among the most suitable tools for in vivo gene delivery due to their superior infection efficiency and unique biological and biophysical properties [22]. AAV shows persistent transgene expression and long-term correction of disease phenotype with little or no toxicity in clinical and preclinical studies [23]. In our current study, we used AAV equipped with a synthetic gene to manipulate immune cells. To the best of our knowledge, this is the first report describing that an AAV carrying a CAR gene can reprogram immune cells in vivo to generate enough CAR T cells to induce tumor regression. Our current CAR gene-carrying AAV strategy contrasts with the costly and time-consuming method of conventional manufacturing CAR T therapy, which requires primary $\mathrm{T}$ cell isolation and transgene introduction and expansion via lentiviral vectors or retroviral vectors ex vivo.

We used humanized NCG-HuPBL mice throughout our study, which not only recapitulate ATL but also other antitumor immunological characteristics [24]. In our current study, we chose CD4targeting CAR transgenes because $\mathrm{CD}^{+}$leukemia and lymphoma are highly aggressive types of tumors that are extremely refractory to chemotherapy. Initial preclinical studies of CAR T cells for targeting $\mathrm{CD}^{+}{ }^{+}$tumors are encouraging, but to date, no standard care has been developed [25]. Furthermore, CD4+ cells are the major latent reservoir of HIV, posing a challenge for HIV eradication; therapies targeting $\mathrm{CD}^{+}$cells can also be translated into HIV treatment [26] and treatments for other infectious diseases, including systemic lupus erythematosus, rheumatoid arthritis, multiple sclerosis and psoriasis [27-30]. Previously, our group tested the same targeting antibody (Hu5A8) in humanized NSG-HuPBL mice for HIV treatment [10]. 
421 An interesting outcome of our study was that AAVs are capable of producing enough in vivo CAR

$422 \mathrm{~T}$ cells upon a single infusion, and these $\mathrm{T}$ cells are potent as ex vivo-generated CAR T cells.

423 Furthermore, once produced, they act as a living drug, are distributed throughout the host, and

424 circulate in the host body for weeks, with the ability to recognize and destroy target cells.

425 Conventional lentivirally or retrovirally transduced cells tend to lose their transgenes expression

426 over time [31]. AAV vectors lack engineered lipids and chemical components and are free from

427 viral genes, and thus considered to be safe for gene therapy with limited toxicity [32]. In general,

428 AAV has been reported to be less immunogenic than other viruses and synthetic carriers.

429 The complete $\mathrm{CD}^{+}{ }^{+}$depletion in NCG-HuPBL mice raises concern for adverse effects in the long

430 term or clinical setting. CD4 helper T cell depletion for long periods leads to "on-target, off-tumor"

431 toxicities and immunodeficiency in patients [33]. Furthermore, the persistence and production of

432 memory AAV-CD4CAR T cells may further extend CD4 cell aplasia in the clinical setting, thereby

433 suggesting the need for a safety switch. One such approach is to eliminate CAR T cells completely

434 from the host. Ma et al. [34] used alemtuzumab (anti-CD52 antibody), which resulted in

435 eliminating CAR T cells within a few hours. Furthermore, such a situation can also be tackled in

436 advance by integrating recently documented multiple advanced logic and control features for CAR

437 T cells $[35,36]$ into our ACG system.

438 CAR T cells have emerged as a promising therapy for the treatment of various tumors during the

439 last few years and have obtained approval from the FDA to treat large B cell lymphomas such as

440 non-Hodgkin lymphoma and B cell ALL [1]. Currently, multiple clinical trials and preclinical

441 studies are ongoing to expand the therapeutic applications of CAR T cells. As mentioned earlier,

442 CAR $\mathrm{T}$ cell production is a multiplex system involving cell therapy, gene therapy, and

443 immunotherapy. This multiplex system of CAR T cell production limits the therapeutic potential 
444 of CAR T cells to a few tumor types and specialized centers around the globe [37]. Several

445 strategies are currently being developed to generate allogenic CAR T cells, including genome

446 editing of T cells [38], in vivo engineering of T cells relying on the infusion of nanoparticles [39],

447 and strategies involving lentivirus [40], using alternative effector cells (NK cells, $\gamma \delta \mathrm{T}$ cells, and

448 macrophages) [41, 42], donor-derived allogeneic CAR T cells [43], and nonalloreactive T cells

449 [44] as well as the split, universal, and programmable CAR system (SUPRA) [35]. All these

450 approaches bear advantages and disadvantages [45]; however, we believe that the AAV-based in

451 vivo approach may be simpler to operate in a clinical setting since it transduces dividing and

452 nondividing cells without requiring a strong $\mathrm{T}$ cell activation signal. Unlike, Lentivirus and

453 synthetic vector, AAV DNA can persist in episomal form and used host endoplasmic reticulum

454 for uncoating. The episomal form of the AAV DNA is very stable and cannot be degraded by

455 exonucleases [46], thus making it a suitable carrier for manipulating in vivo cells. Additionally,

456 ample evidence from various AAV-based clinical studies has shown the safety of AAVs delivering

457 genes [47].

458 Our ACG strategy involving in vivo generation of CAR T cells has the potential to overcome the

459 abovementioned hurdles and will make CAR T cell treatment easier and affordable, and will

460 transform an intricate, individualized treatment into a broadly applicable product. AAV-DJ

461 subtype for manipulating in vivo immune cells exhibits higher transduction efficiency and broader

462 tropism [11, 48]. Therefore, upon infusion in humanized mice, AAV-CD4 CAR based on AAV-

463 DJ subtype might infect numerous host and target cells. However, the current study aims to give a

464 proof of concept of the ACG method; thus, we evaluate the in vivo generation and properties of

465 key effector cells for CAR therapy, i.e., T cells. Despite our strong proof-of-concept of ACG, there

466 are still many concerns and limitations in our current study, which need to be addressed in the 
467 preclinical setting to make these in vivo-generated AAV-CAR T cells as effective and safe as ex

468 vivo-generated CAR T cells. One major limitation of the current study is the specificity of AAV

469 carrying the CAR gene, and we did not evaluate the CAR expression in hematopoietic cells, tumor

470 cells, and other immune cells. Furthermore, the current study is the lack of having strong data to

471 evaluate the biocompatibility and safety of the ACG approach, and the Hu-PBL NCG may not

472 fully recapitulate the human immune microenvironment. Further studies will be required to design

473 an ACG with ligand coupling or modification in the AAV capsid for cell-specific delivery of the

474 CAR gene. Additionally, a deep insight into its biocompatibility and safety in larger and versatile

475 animal models, and evaluating its therapeutic potential in other tumor models will facilitate clinical

476 translation of this approach. In addition to the in vivo CAR $\mathrm{T}$ cells generation, given further

477 optimization, our ACG strategy may lay the foundation for in vivo $\mathrm{T}$ cell manipulation in

478 immunotherapy beyond cancer and infectious diseases. We believe that the ACG strategy will

479 substantially streamline the manufacturing process of cell-based therapies in the clinical setting

480 and will make genetically engineered cell treatment less expensive and more effective.

484 The patent (CN201910224056.8) was filed by Xilin Wu, Zhiwei Wu and Waqas Nawaz as

485 inventors of the AAV in vivo-generated CAR cell concept. All other authors declare that they

486 have no conflicts of interest.

\section{$487 \quad$ Findings}

489 This work was supported by the National Science Foundation of China (NSFC) (No. 81803414,

490 31970149), The Major Research and Development Project (2018ZX10301406), Jiangsu Province

491 Natural Science Foundation for Young Scholar (grant \#BK20170653), Nanjing University- 
492 Ningxia University Collaborative Project (grant \#2017BN04), Jiangsu Province

493 "Innovative and Entrepreneurial Talent” project and Six Talent Peaks Project of Jiangsu Province.

494

495 Data availability

496 Plasmids generated in this study will be made available upon reasonable request from the

497 corresponding author. Detailed methods, materials, and troubleshooting strategies are provided as

498 Supplementary Information.

$500 \quad$ Ethics

501 The study and the protocol for this research were approved by the Center for Public Health

502 Research, Medical School, Nanjing University. All experimental animal procedures were 503 approved by the Committee on the Use of Live Animals by the Ethics Committee of Nanjing Drum

504 Tower Hospital. All the authors declare their compliance with publishing ethics.

505

506 Author contribution

507 All authors contributed to the work fulfilling the criteria adopted from ICMJE. Acquisition of data:

508 WN, WX, BH, SX, ZR, ZL, and YL. Analysis and interpretation of data: WN, WX, SX, and YL.

509 Drafting of the manuscript: WN, WX, and WZ. Critical revision: WX and WZ. Study conception

510 and design: WX and WZ. Financial support: WX and WZ. All authors read and approved the

511 submitted version of the manuscript. Each author has agreed both be personally accountable for

512 the author's own contributions and to ensure that questions related to the accuracy or integrity of

513 any part of the work, even those in which the author was not personally involved, are appropriately

514 investigated and resolved and that the resolution is documented in the literature. 


\section{References}

1. Lichtman, E.I. and G. Dotti, Chimeric antigen receptor T-cells for B-cell malignancies. Transl Res, 2017. 187: p. 59-82.

2. Yip, A. and R.M. Webster, The market for chimeric antigen receptor $T$ cell therapies. Nat Rev Drug Discov, 2018. 17(3): p. 161-162.

3. Levine, B.L., et al., Global Manufacturing of CAR T Cell Therapy. Mol Ther Methods Clin Dev, 2017. 4: p. 92-101.

4. Torikai, H. and L.J. Cooper, Translational Implications for Off-the-shelf Immune Cells Expressing Chimeric Antigen Receptors. Mol Ther, 2016. 24(7): p. 1178-86.

5. $\quad$ Roddie, C., et al., Manufacturing chimeric antigen receptor T cells: issues and challenges. Cytotherapy, 2019. 21(3): p. 327-340.

6. Hamieh, M., et al., CAR T cell trogocytosis and cooperative killing regulate tumour antigen escape. Nature, 2019. 568(7750): p. 112-116.

7. https://clinicaltrials.gov/. AAV. 2020, january 31; Available from: https://clinicaltrials.gov/ct2/results/browse?term=AAV\&brwse=cond_alpha_all.

8. Wang, D., P.W.L. Tai, and G. Gao, Adeno-associated virus vector as a platform for gene therapy delivery. Nat Rev Drug Discov, 2019.

9. Grimm, D., et al., In Vitro and In Vivo Gene Therapy Vector Evolution via Multispecies Interbreeding and Retargeting of Adeno-Associated Viruses. Journal of Virology, 2008. 82(12): p. 5887.

10. Wu, X., et al., Tandem bispecific neutralizing antibody eliminates HIV-1 infection in humanized mice. J Clin Invest, 2018. 128(6): p. 2239-2251.

11. Kimura, T., et al., Production of adeno-associated virus vectors for in vitro and in vivo applications. Sci Rep, 2019. 9(1): p. 13601.

12. Kochenderfer, J.N., et al., Construction and preclinical evaluation of an anti-CD19 chimeric antigen receptor. J Immunother, 2009. 32(7): p. 689-702.

13. Chen, Y.H., M.S. Keiser, and B.L. Davidson, Adeno-Associated Virus Production, Purification, and Titering. Curr Protoc Mouse Biol, 2018. 8(4): p. e56.

14. Ellis, B.L., et al., A survey of ex vivo/in vitro transduction efficiency of mammalian primary cells and cell lines with Nine natural adeno-associated virus (AAV1-9) and one engineered adeno-associated virus serotype. Virology journal, 2013. 10: p. 74-74.

15. Ihsan, A.U., et al., Establishment of a rat model of chronic Prostatitis/Chronic Pelvic Pain Syndrome (CP/CPPS) induced by immunization with a novel peptide T2. Biomed Pharmacother, 2017. 91: p. 687-692.

16. $\mathrm{Wu}, \mathrm{X}$., et al., A single-domain antibody inhibits SFTSV and mitigates virus-induced pathogenesis in vivo. JCI Insight, 2020. 5(13).

17. Davenport, A.J., et al., Chimeric antigen receptor T cells form nonclassical and potent immune synapses driving rapid cytotoxicity. Proc Natl Acad Sci U S A, 2018. 115(9): p. E2068-e2076.

18. Shu, S.T., et al., A novel bioluminescent mouse model and effective therapy for adult Tcell leukemia/lymphoma. Cancer Res, 2007. 67(24): p. 11859-66.

19. Yu, D., et al., Targeting Jurkat T Lymphocyte Leukemia Cells by an Engineered Interferon-Alpha Hybrid Molecule. Cell Physiol Biochem, 2017. 42(2): p. 519-529.

20. Matsui, W., et al., Characterization of clonogenic multiple myeloma cells. Blood, 2004. 103(6): p. 2332-2336. 
21. Grupp, S.A., et al., Chimeric antigen receptor-modified T cells for acute lymphoid leukemia. N Engl J Med, 2013. 368(16): p. 1509-18.

22. Mingozzi, F. and K.A. High, Therapeutic in vivo gene transfer for genetic disease using $A A V$ : progress and challenges. Nature Reviews Genetics, 2011. 12: p. 341.

23. Brady, J.M., D. Baltimore, and A.B. Balazs, Antibody gene transfer with adenoassociated viral vectors as a method for HIV prevention. Immunological reviews, 2017. 275(1): p. 324-333.

24. Wu, Y. and X.-Z. Yu, Modelling CAR-T therapy in humanized mice. EBioMedicine, 2019. 40: p. 25-26.

25. Pinz, K., et al., Preclinical targeting of human T-cell malignancies using CD4-specific chimeric antigen receptor (CAR)-engineered T cells. Leukemia, 2016. 30(3): p. 701-707.

26. Emu, B., et al., Phase 3 Study of Ibalizumab for Multidrug-Resistant HIV-1. N Engl J Med, 2018. 379(7): p. 645-654.

27. Isaacs, J.D., et al., Humanized anti-CD4 monoclonal antibody therapy of autoimmune and inflammatory disease. Clinical and experimental immunology, 1997. 110(2): p. 158166.

28. van Oosten, B.W., et al., Treatment of multiple sclerosis with the monoclonal anti-CD4 antibody cM-T412: results of a randomized, double-blind, placebo-controlled, MRmonitored phase II trial. Neurology, 1997. 49(2): p. 351-7.

29. Morel, P., et al., Anti-CD4 monoclonal antibody therapy in severe psoriasis. J Autoimmun, 1992. 5(4): p. 465-77.

30. Pohlers, D., et al., Anti-CD4 monoclonal antibody treatment in acute and early chronic antigen-induced arthritis: influence on Thelper cell activation. Clin Exp Immunol, 2004. 135(3): p. 409-15.

31. Ellis, J., Silencing and variegation of gammaretrovirus and lentivirus vectors. Hum Gene Ther, 2005. 16(11): p. 1241-6.

32. Naso, M.F., et al., Adeno-Associated Virus (AAV) as a Vector for Gene Therapy. BioDrugs : clinical immunotherapeutics, biopharmaceuticals and gene therapy, 2017. 31(4): p. 317-334.

33. Luckheeram, R.V., et al., $C D 4^{+}$T cells: differentiation and functions. Clinical \& developmental immunology, 2012. 2012: p. 925135-925135.

34. Ma, G., et al., Targeting T Cell Malignancies Using CD4CAR T-Cells and Implementing a Natural Safety Switch. Stem Cell Rev Rep, 2019. 15(3): p. 443-447.

35. Cho, J.H., J.J. Collins, and W.W. Wong, Universal Chimeric Antigen Receptors for Multiplexed and Logical Control of T Cell Responses. Cell, 2018. 173(6): p. 14261438.e11.

36. Zhao, J., et al., Universal CARs, universal T cells, and universal CAR T cells. Journal of hematology \& oncology, 2018. 11(1): p. 132-132.

37. Wang, X. and I. Rivière, Clinical manufacturing of CAR T cells: foundation of a promising therapy. Molecular Therapy - Oncolytics, 2016. 3: p. 16015.

38. Qasim, W., et al., Molecular remission of infant B-ALL after infusion of universal TALEN gene-edited CAR T cells. Sci Transl Med, 2017. 9(374).

39. Smith, T.T., et al., In situ programming of leukaemia-specific T cells using synthetic DNA nanocarriers. Nature Nanotechnology, 2017. 12: p. 813. 
606

607

608

609

610

611

612

613

614

615

616

617

618

619

620

621

622

623

624

625

626

627

628

629

630

631

632

633

40. Pfeiffer, A., et al., In vivo generation of human CD19-CAR T cells results in B-cell depletion and signs of cytokine release syndrome. EMBO Molecular Medicine, 2018. 10(11): p. e9158.

41. Klichinsky, M., et al., Abstract 4575: Chimeric antigen receptor macrophages (CARMA) for adoptive cellular immunotherapy of solid tumors. Cancer Research, 2017. 77(13 Supplement): p. 4575.

42. Romanski, A., et al., CD19-CAR engineered NK-92 cells are sufficient to overcome NK cell resistance in B-cell malignancies. J Cell Mol Med, 2016. 20(7): p. 1287-94.

43. Ghosh, A., et al., Donor CD19 CAR T cells exert potent graft-versus-lymphoma activity with diminished graft-versus-host activity. Nat Med, 2017. 23(2): p. 242-249.

44. Chan, W.K., et al., Chimeric antigen receptor-redirected CD45RA-negative T cells have potent antileukemia and pathogen memory response without graft-versus-host activity. Leukemia, 2015. 29(2): p. 387-95.

45. Depil, S., et al., 'Off-the-shelf' allogeneic CAR T cells: development and challenges. Nat

46. Rossi, A., et al., Vector uncoating limits adeno-associated viral vector-mediated transduction of human dendritic cells and vector immunogenicity. Sci Rep, 2019. 9(1): p. 3631.

47. Verdera, H.C., K. Kuranda, and F. Mingozzi, AAV Vector Immunogenicity in Humans: A Long Journey to Successful Gene Transfer. Mol Ther, 2020.

48. Jollé, C., et al., Development of Efficient AAV2/DJ-Based Viral Vectors to Selectively Downregulate the Expression of Neuronal or Astrocytic Target Proteins in the Rat Central Nervous System. 2019. 12(201).

\section{Figures and figure legends:}


a

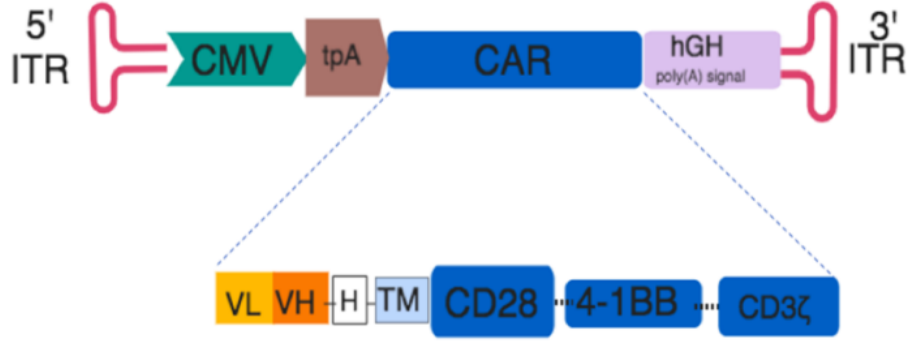

ScFv (Targeting domain of CAR, either Hu5a8 or Rit)

Hinge/Spacer

Transmembrane domain

Signaling domains b $\mathrm{KDa}$

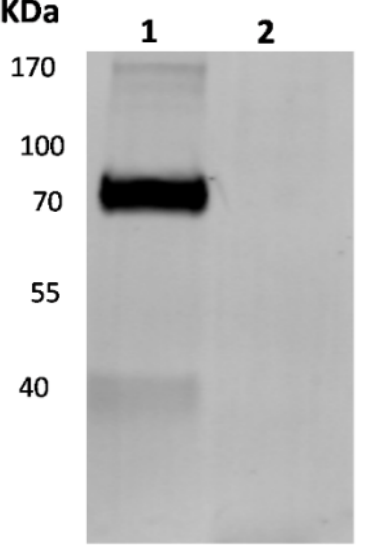

AAV-CD4CAR (H)
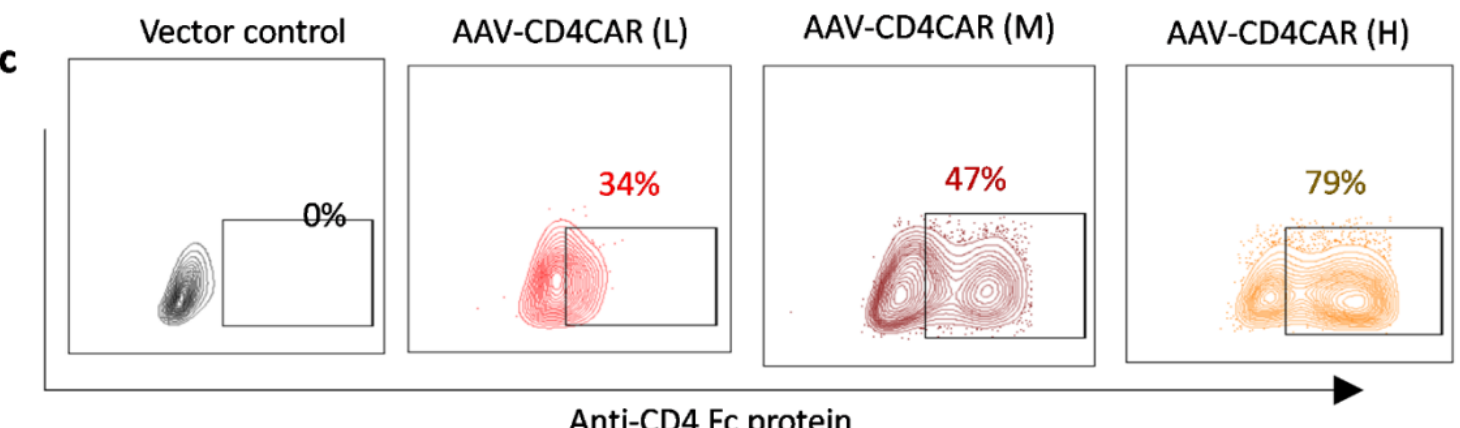

Anti-CD4 Fc protein

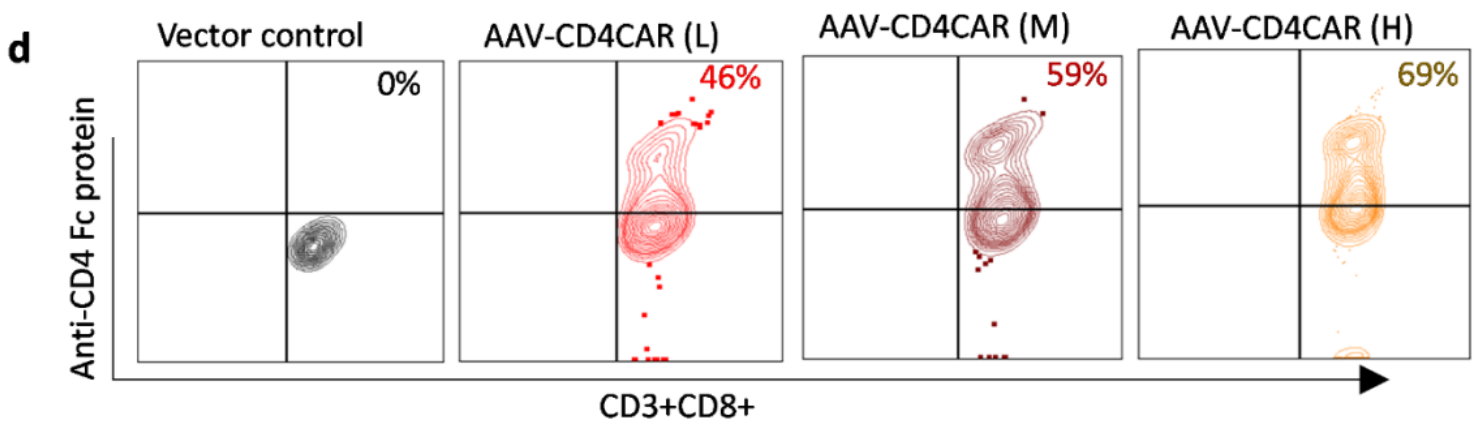

Figure 1. AAV-CD4CAR construct and characterization (a) Schematic representation of the AAV encoding CD4CAR or CD20CAR. The third generation of the AAV-CAR contains a leader sequence, the anti-CD4 (Hu5a8) or the anti-CD20 scFv region (Rit), a hinge domain (H), a transmembrane domain (TM) and intracellular signaling domains from the following molecules: CD28 (coactivator), 4-1BB (coactivator), and CD3 zeta. (b) HEK293T cells were infected with AAV-CD4CAR (lane 1) and AAV-GFP (lane 2) for Western blot analysis, and at 48 hours posttransduction, they were probed with mouse anti-human CD3 zeta antibody, with the CD4-CAR band observed at the expected size $(\sim 75 \mathrm{kDa})$ and a breakdown product observed at $\sim 37 \mathrm{kDa}$. 
644 However, no band was observed for AAV-GFP-transduced cells. (c) HEK293T cells were infected 645 with different doses of AAV-CD4CAR. 48 hours post-infection, cells were stained with CD4-rFc 646 protein followed by secondary antibody staining with Alexa Fluor 488-conjugated goat anti-rabbit $647 \mathrm{IgG}(\mathrm{H}+\mathrm{L})$. (d) AAV-CD4 CAR expression on CD3+CD+8 cells. Activated PBMCs were infected 648 with different doses of AAV-CD4CAR or uninfected (vector control). Forty-eight hours post649 infection, cells were stained with CD4-rFc protein for CAR expression, followed by secondary 650 antibody staining with Alexa-Fluor 488-conjugated goat anti-rabbit IgG $(\mathrm{H}+\mathrm{L}) .1 \times 10^{4}$ AAVs/cell 651 was considered AAV low (L), $1.5 \times 10^{4}$ AAVs/cell was considered AAV middle (M), and $1 \times 10^{5}$ 652 AAVs/cell was considered AAV high $(\mathrm{H})$. 
a
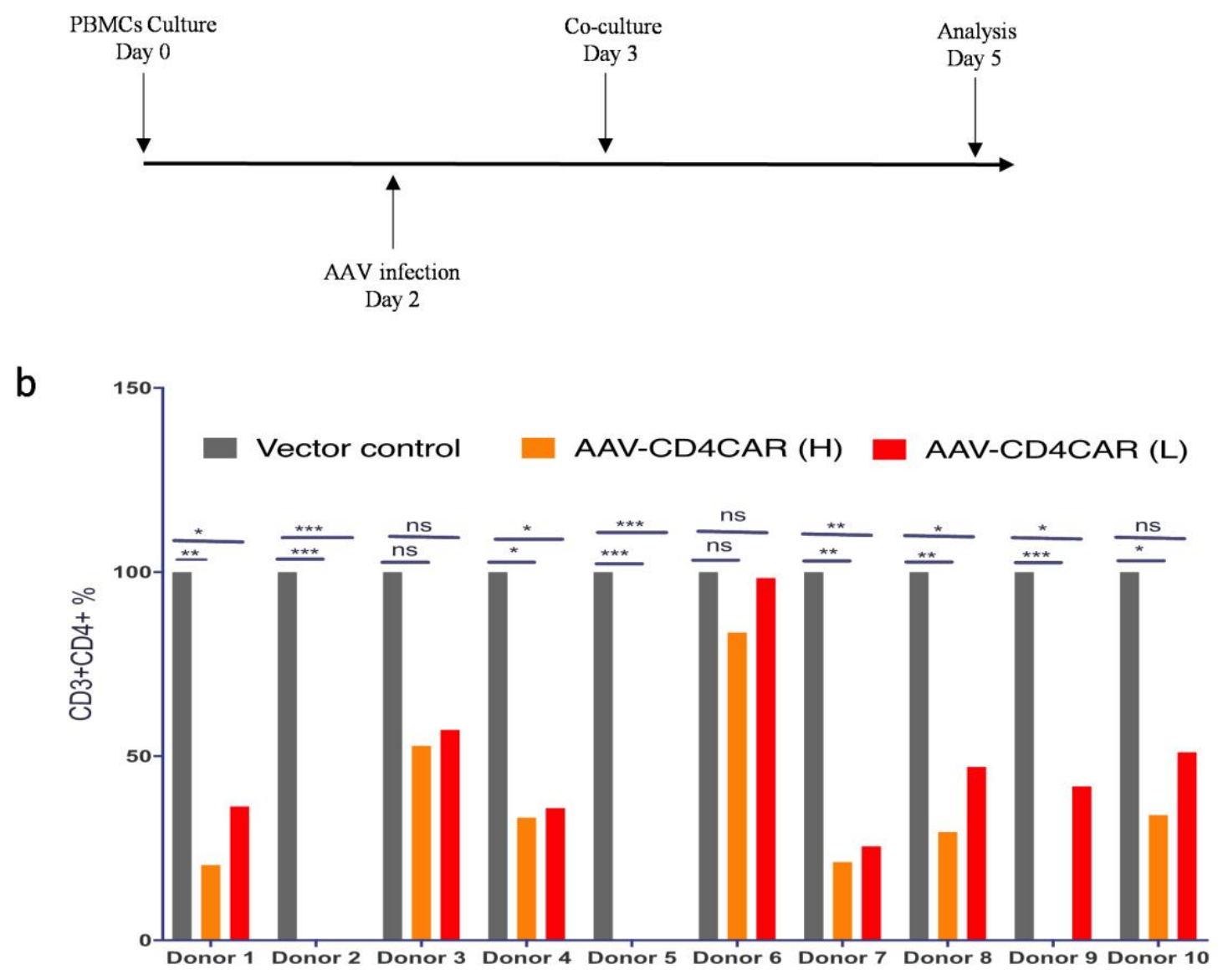

C

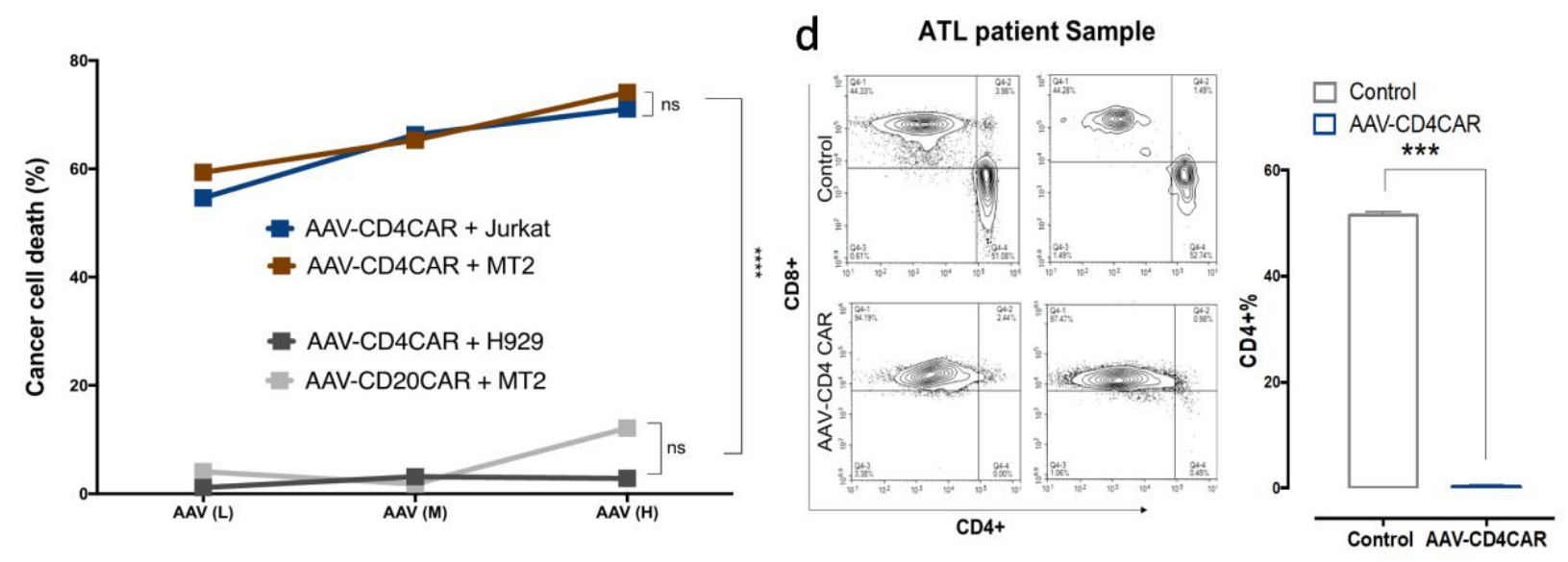

Figure 2. AAV-CD4CAR induces antitumor activity (a) Schematic representation showing the ex vivo AAV-CAR production and co-culture assays. (b) AAV-CD4CAR depleted CD3+CD4+ cells in different donors $(\mathrm{n}=10)$ during the course of T cell expansion. Activated PBMCs from each donor were transduced with different doses of AAV-CD4CAR, and Vector control (untransduced). After 48 hours, the cells were analyzed by FACS using anti-human CD4, anti-human CD3, and anti-human CD8 antibodies. The CD3+CD4+ percentage was normalized to the vector control 
664 group (which represented 100\%), and the CD3+CD4+ percentages of AAV-CD4CAR (H) and 665 AAV-CD4CAR (L) was calculated by the percent difference from the vector control group. A two666 way ANOVA multiple comparisons test within each donor was used for statistical analysis. ns, no 667 significance; ***, $\mathrm{P}<0.001 ; * *, \mathrm{P}<0.01$; *, $\mathrm{P}<0.05$. (c) AAV-CD4CAR T cells were co-cultured 668 with CFSE-stained target cells, 48 hours post-co-culture, cells were stained with 7AAD and 669 analyzed by FACS. Two-way ANOVA with the multiple-comparisons test was used to assess 670 significance, ns, no significance; **** $\mathrm{P}<0.0001$. ***, $\mathrm{P}<0.001 ; * *, \mathrm{P}<0.01 ; *, \mathrm{P}<0.05$. (d) 671 ATL patient PBMCs were infected with AAV-CD4CAR $(\mathrm{H})$ and with a control vector (samples 672 were duplicated). 48 hours post-transduction, cells were analyzed by FACS for CD3+CD4+ 673 depletion. An unpaired two-tailed t-test was used to calculate the significance of the difference.

674 AAV-CD4CAR vs vector control, ***, $\mathrm{P}<0.0001$.

675

676

677 
a

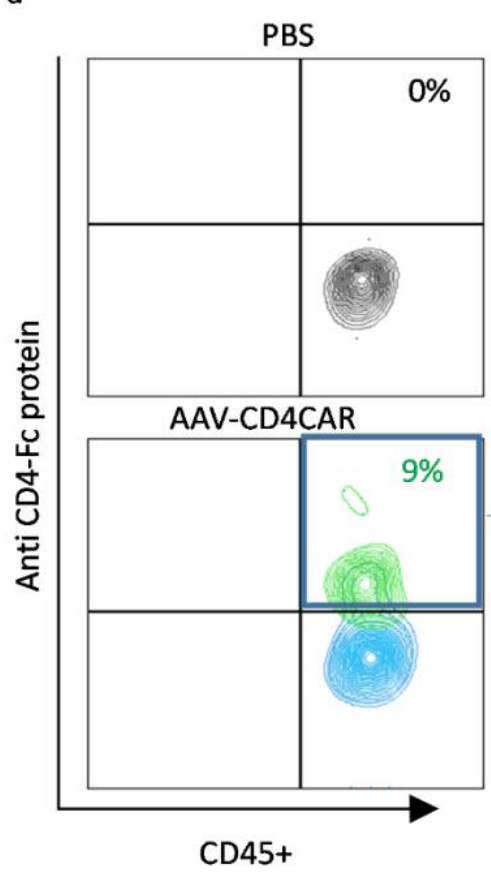

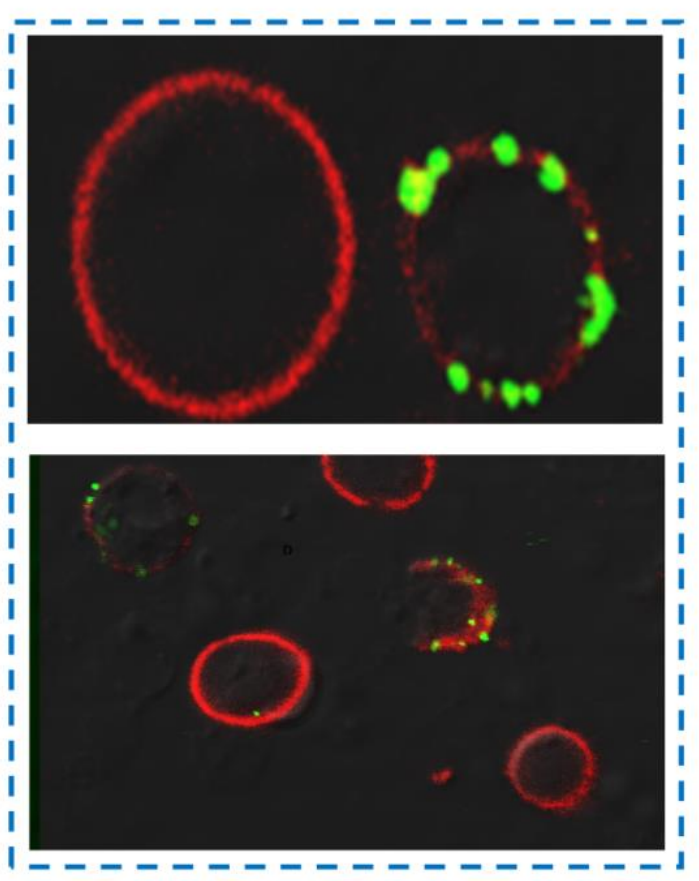

C

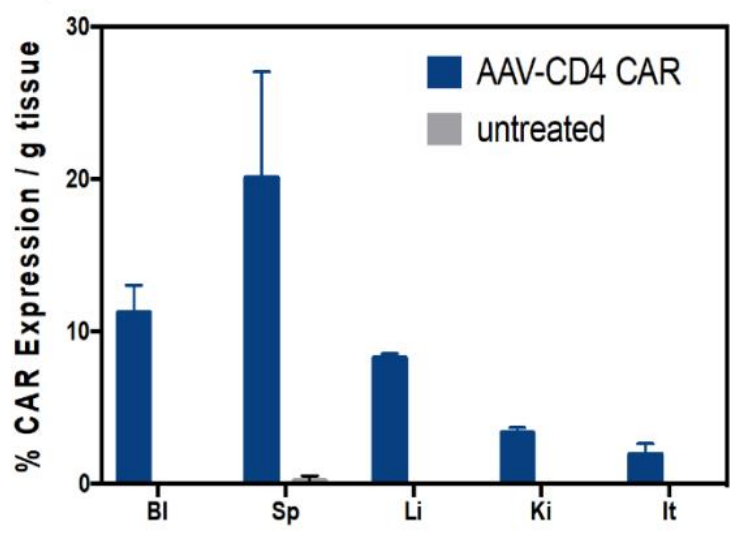

d

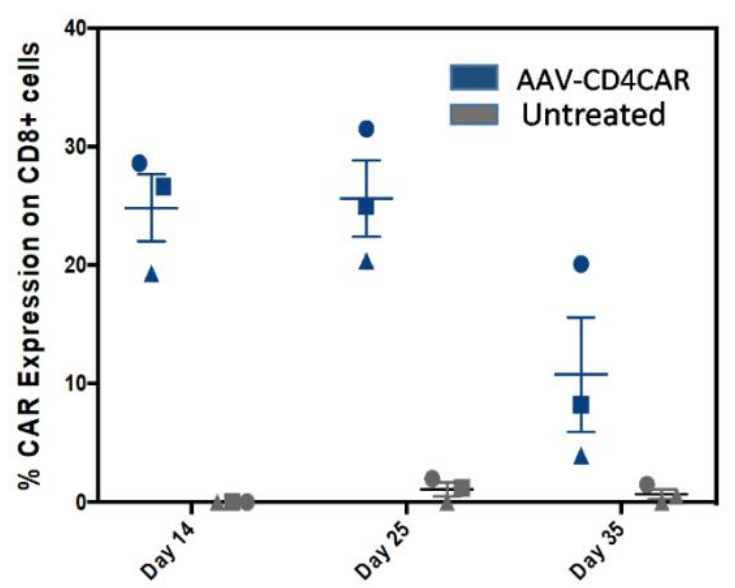

AAV-CD4CAR
Untreated
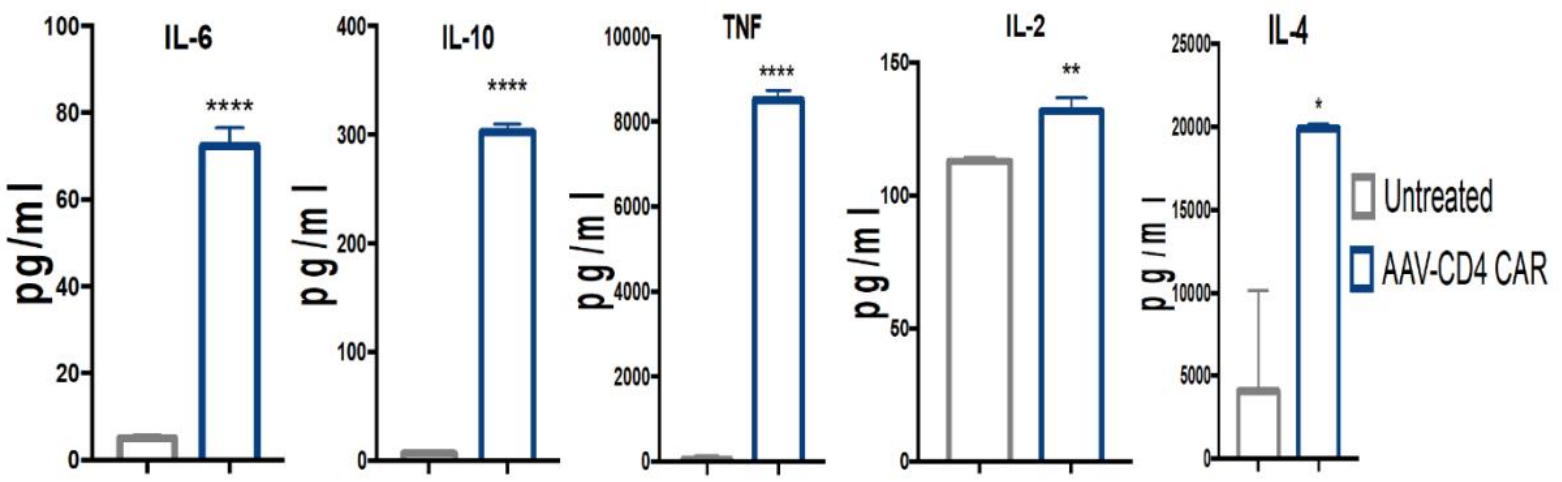
679 Figure 3. AAV-CD4CAR reprograms immune cells and generates in vivo tumor-specific 680 AAV-CD4CAR T cells. (a) Representative flow cytometry results showing the expression of 681 AAV-CD4CAR on CD45+ cells 48 hours after infusing a single dose of $1 \times 10^{11}$ AAV-CD4CAR 682 into NCG-HuPBL mice $(n=3)$. The right panel shows the confocal imaging of CD45+ cells 683 expressing AAV-CD4CAR expression. The red shows the cellular membrane, whereas the green 684 dots represent AAV-CD4CAR expression on the cell. (b) A total of $1 \times 10^{11}$ AAVs carrying the 685 CD4CAR gene were injected into mice $(n=3)$, and AAV-CD4CAR expression was demonstrated by FACS at the indicated time. Distinct symbols represent different donor PBMCs used for the development of the HuNCG mouse model. All data are shown as the mean \pm s.e.m. with error bars on the graph. (c) Graphical representation of the biodistribution of AAV-CD4CAR T cells in various tissues $(\mathrm{n}=3)$. Bl, blood; Sp, spleen; Li, liver; Lu, lung; It, intestine; Ki, kidney. Cells isolated from the spleen, liver, kidney, blood, and intestine were analyzed by flow cytometry for AAV-CD4CAR expression on CD8+ cells. All data are shown as the mean \pm s.e.m. with error bars on the graph. (d) Graphical representation of different cytokines detected in the plasma of tumorbearing ATL NCG-HuPBL mice $(n=3)$, at 2 weeks after AAV injection. Cytokines were detected through FACS by using the BD Cytometric Beads Array Human Th1/Th2 Cytokine Kit. An unpaired two-tailed t-test was used for analysis. ns, no significance; ****P $<0.0001$. ***, $\mathrm{P}<$ $0.001 ; * *, \mathrm{P}<0.01 ; *, \mathrm{P}<0.05$. 
a

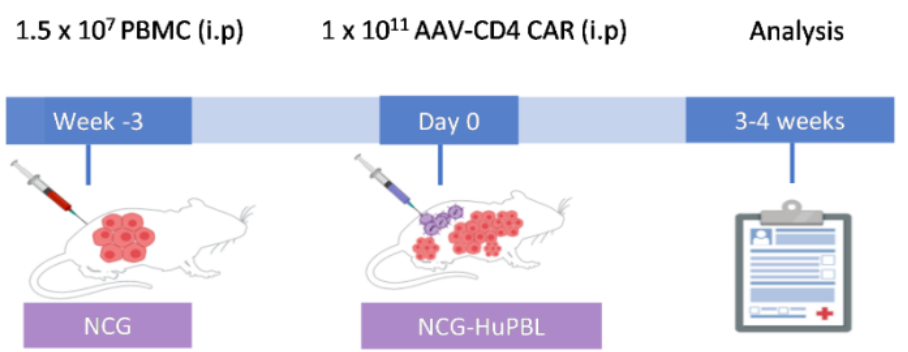

b
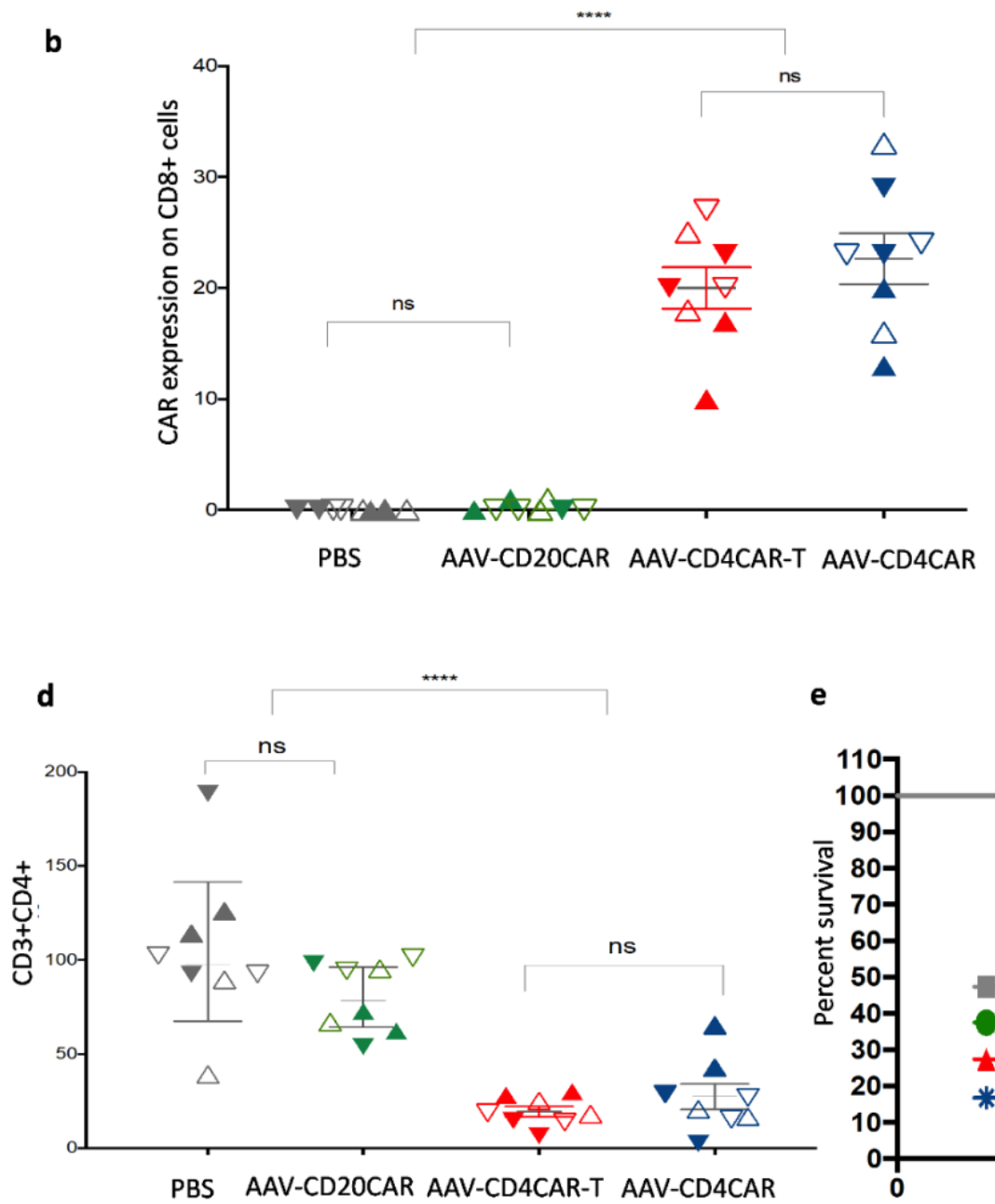

e

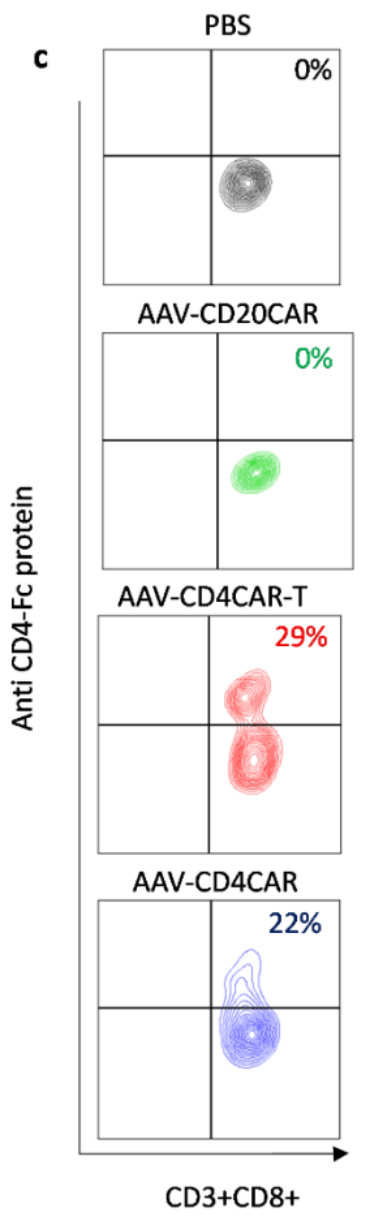

Figure 4. Functional characterization and comparative study of in vivo-generated AAVCD4CAR T cells. (a) Schematic representation showing the development of NCG-HuPBL mouse model and experimental design. (b) Graphical representation of CAR expression using the CD4Fc protein in different groups one week after the treatment. The NCG-HuPBL mouse model was developed by using PBMCs from four different healthy donors. Successful NCG-HuPBL mice were randomly divided into four groups, and each group contained eight mice from four different donors. Distinct symbols are used to differentiate the NCG-HuPBL mice engrafted with different 
711 text. Blood was collected from each mouse and evaluated for CAR expression through FACS. 712 Data were analyzed by using two-way ANOVA for multiple comparisons. $* * * * \mathrm{P}<0.0001$, ns,

713 No significance. (c) Exemplary counter plot showing the CAR expression of a single mouse 714 sample from each group. (d) Graphical representation of the normalized CD3+CD4+ percentage 715 documented after 14 days of treatment. Two weeks following the treatment, peripheral blood was 716 collected from each mouse and analyzed for the CD3+CD4+ population through FACS. The $717 \mathrm{CD} 3+\mathrm{CD} 4+$ cell percentage of each mouse was normalized to $100 \%$ at day 0 . The CD3+CD4+ 718 percentage at week 2 for each mouse was calculated by the percent difference from the 719 corresponding mice at day 0. Data were analyzed by using two-way ANOVA for multiple 720 comparisons. $* * * * \mathrm{P}<0.0001$, ns, No significance. (e) Kaplan-Meier curves for percent survival 721 of mice used in (A and B). Percent survival between the groups was analyzed by the log-rank test; $722 \quad \mathrm{P}<0.05$ was considered significant. 

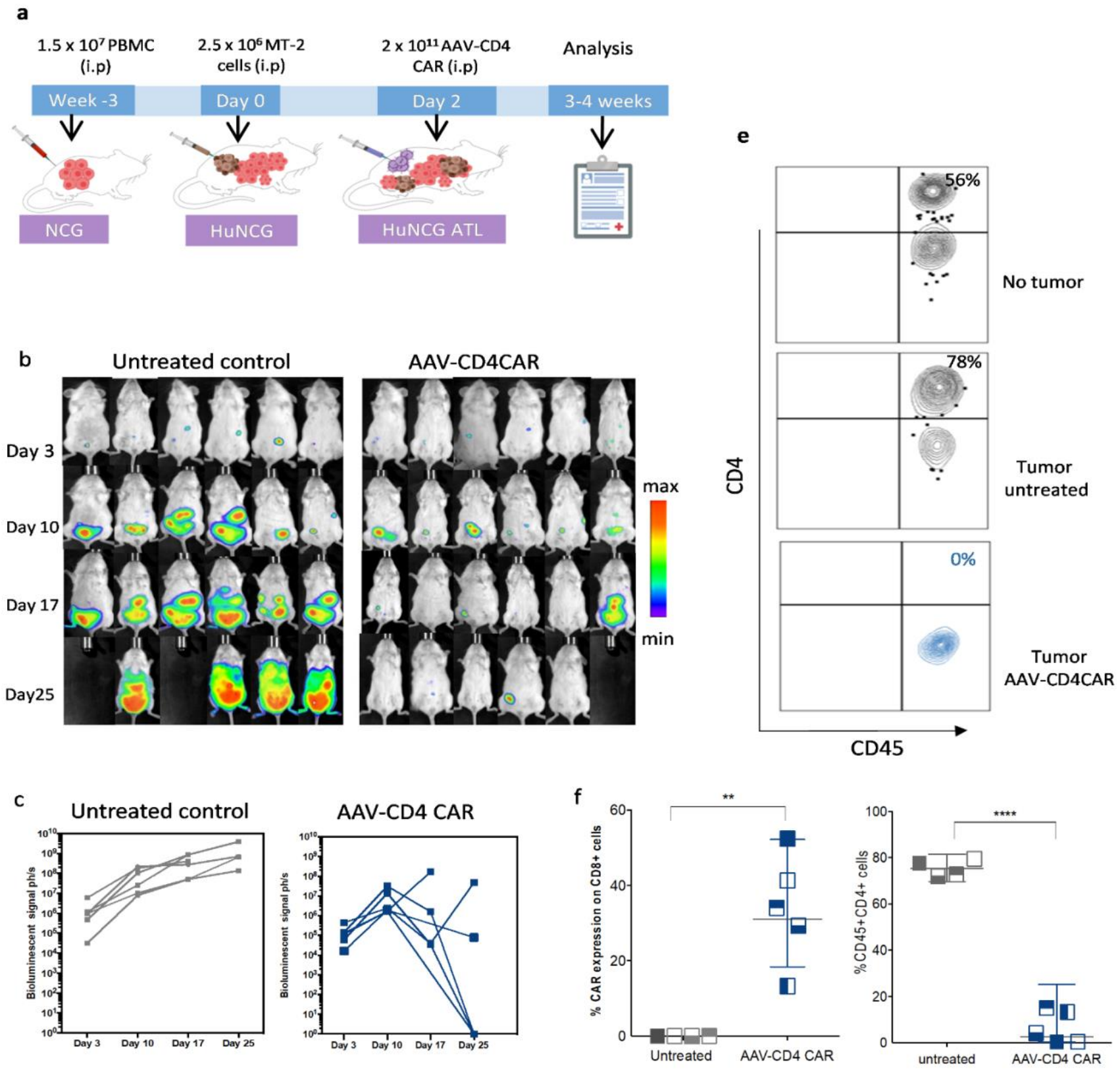

d

g
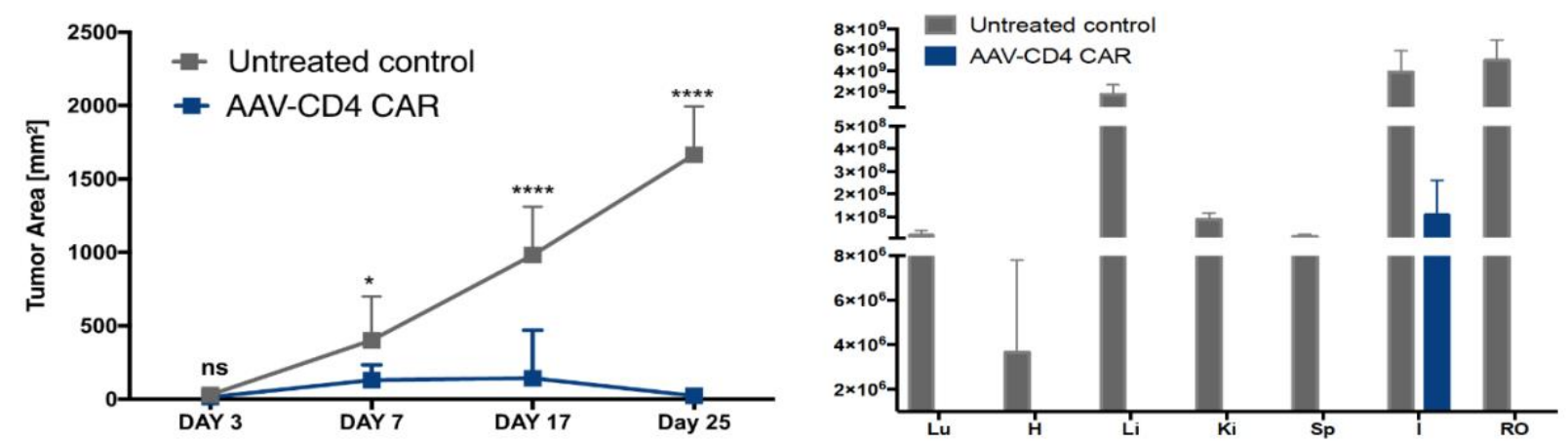
Figure 5. In vivo-generated AAV-CD4CAR T cells mediated antitumor activity. (a) Schematic representation showing the development of HuNCG ATL mouse model and experimental design. (b) Sequential in vivo imaging of luciferase-expressing MT2 ATL cells injected into NCG-HuPBL mice. Following AAV or PBS (untreated) injection, mice were imaged the next day, followed by in vivo imaging at different time points as specified in the figure. Blank images represent dead mice. (c) Bioluminescent intensity (p/sec) of mice shown in B. Each line represents one mouse. (d) Mean tumor area of AAV-CD4CAR-treated mice and untreated mice. Two-way ANOVA with multiple comparisons was used to analyze the mean tumor area. ns, no significance , $* \mathrm{P}<0.05$, $* * * * \mathrm{P}<0.0001$. Data are presented as the mean \pm SEM. (e) Exemplary counterplot of FACS analysis showing the CD45+CD4+ percentage in NCG-HuPBL mice (no tumor, no treatment), untreated NCG-HuPBL tumor challenge mouse and AAV-CD4CAR-treated NCG-HuPBL tumor challenge mouse at the end of the study. (f) The CD45+CD4+ percentage and AAV-CD4 CAR expression of the mice in B. Blood was collected from each mouse at the end of the study and analyzed by FACS for the CD45+CD4+ percentage and CAR expression on CD3+CD8+ cells. An unpaired t-test was used to analyze the data. **P<0.001, ****P $<0.0001$. (g) Graphical representation of tumor distribution in different organs. Two mice from each group were selected and sacrificed at the end of the study (Supplemental Figure 4). Organs from dissected mice were imaged immediately after sacrifice. The bioluminescent intensity $(\mathrm{pH} / \mathrm{s})$ of each organ was quantified and presented as the mean \pm SEM. Lu, Lung; H, Heart; Li, Liver; Ki, Kidneys; Sp, spleen; I, Large and small intestine; RO, Reproductive organs. 